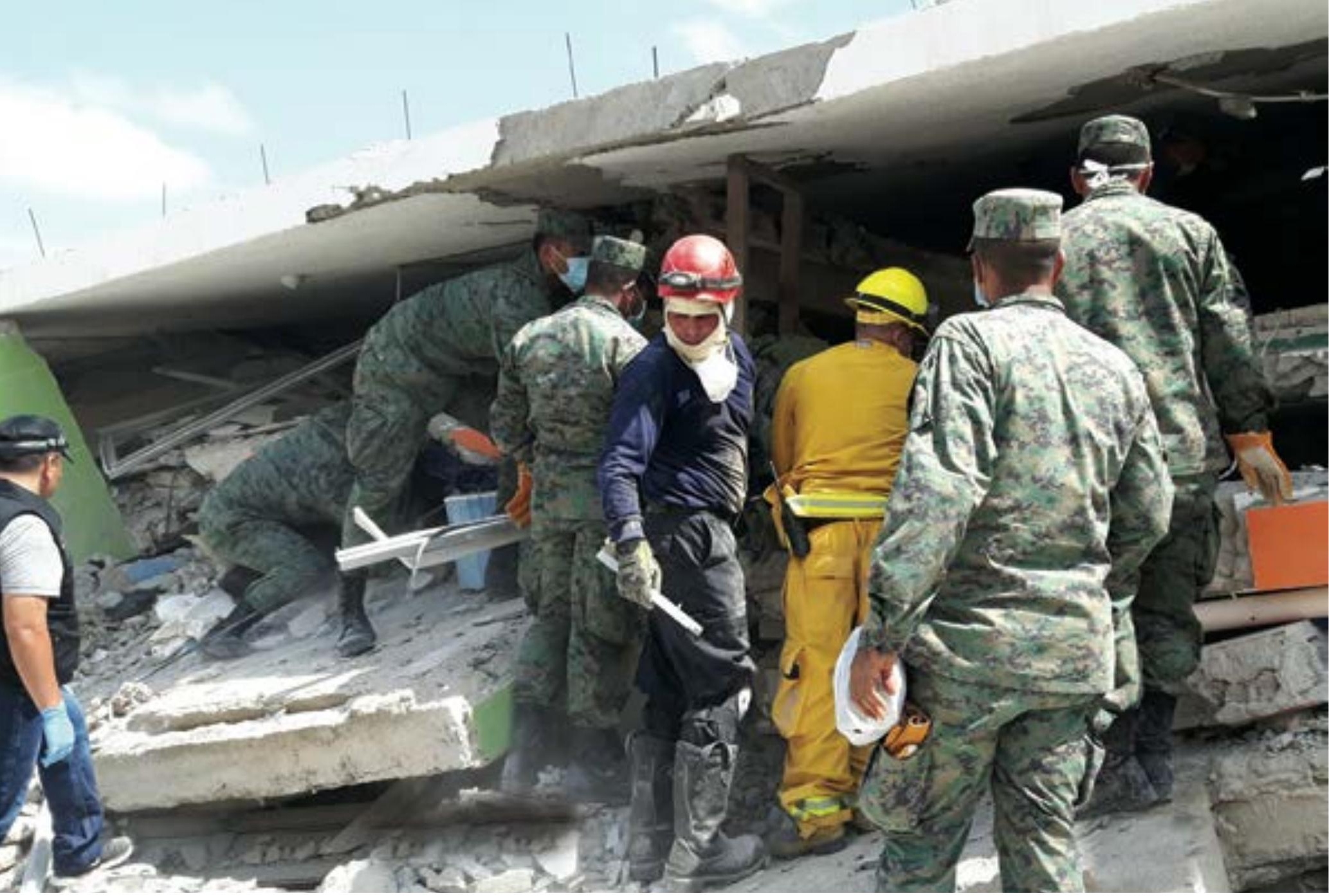

\title{
EL EJÉRCITO ECUATORIANO ANTE SITUACIONES DE EMERGENCIA EN EL TERRITORIO NACIONAL
}

Mayo de A Santiago Villavicencio Silva

\section{RESUMEN}

El planeta sufre cada día las consecuencias y efectos de los eventos de origen natural y antrópico, estos se han constituido en un tema de suma importancia para ser tratados por parte de los Estados que ven la necesidad de plantearse estrategias que se orienten a prevenir, reducir, mitigar y responder frente a estas situaciones.

Es por lo tanto que se requiere que los Estados asuman con responsabilidad la tarea de intervención con sus instituciones de una forma planificada y articulada, potenciando sus capacidades conjuntamente con el sector privado, que les permita actuar con antelación y responder de forma eficaz ante estas necesidades.

Ecuador no ha sido ajeno a esta realidad y como parte de la política de Seguridad Integral donde Fuerzas Armadas (FF.AA.) se encuentra inmerso en apoyo al Servicio Nacional de Gestión de Riesgos y Emergencias (SNGRE), sobre la base de sus capacidades operativas y logísticas, y fundamentada en su estructura organizacional como principal institución de respuesta frente a situaciones de emergencia.

En razón de lo señalado, este artículo tiene la intención de resaltar la intervención que ha asumido el ejército ecuatoriano, en apoyo al Estado y a otras instituciones frente a la ocurrencia de eventos naturales y antrópicos.

Palabras claves: Evento adverso, antrópicos, hábitat, seguridad integral, gestión de riesgos. 


\begin{abstract}
The planet suffers every day the consequences and effects of events of natural and anthropic origin, these have become an issue of utmost importance to be treated by the States that see the need to consider strategies that are oriented to prevent, reduce, mitigate and respond to these situations.

Therefore, it is required that States assume responsibility for the task of intervention with their institutions in a planned and articulated manner, strengthening their capacities jointly with the private sector, enabling them to act in advance and respond effectively to these needs. .

Ecuador has not been oblivious to this reality and as part of the Comprehensive Security policy where the Armed Forces (FF.AA.) is immersed in support of the National Service of Risk and Emergency Management (SNGRE), based on its capabilities operational and logistical, and based on its organizational structure as the main emergency response institution.
\end{abstract}

In view of the above, this article intends to highlight the intervention that the Ecuadorian army has assumed, in support of the State and other institutions in the face of the occurrence of natural and anthropic events.

Keywords: Adverse event, anthropic, habitat, integral security, risk management.

\title{
RESUMO
}

O planeta sofre diariamente as conseqüências e efeitos dos eventos de origem natural e antropogênica, estes tornaram-se uma questão importante a ser abordado pelos Estados que vêem a necessidade de considerar as estratégias que visam prevenir, reduzir, atenuar e responder a essas situações.

É necessário, portanto que os Estados tomem responsabilidade a tarefa de intervenção com as suas instituições de forma planejada e coordenada, reforçar as suas capacidades em conjunto com o setor privado para que possam agir mais cedo e responder eficazmente a essas necessidades .

Equador não foi alheio a esta realidade e como parte da política de segurança abrangente que Fuerzas Armadas (Forças armadas) está imerso em apoio à Gestão de Serviços de Risco Nacional e Emergência (sngre), com base em suas habilidades operacional e logística, e com base em sua estrutura organizacional como a principal instituição de resposta a emergências.

Diante do exposto, este artigo pretende ressaltar a intervenção que o Exército equatoriano assumiu, em apoio ao Estado e a outras instituições frente à ocorrência de eventos naturais e antrópicos.

Palavras chaves: Evento adverso, antrópico, habitat, segurança integral, gerenciamento de risco.

En las últimas décadas el planeta ha vuelto a sufrir los estragos y catástrofes producto de los fenómenos de origen natural y antrópico, tanto los seres humanos como las estructuras han sido sus víctimas, la situación ambiental del planeta se torna preocupante ya que se ve relacionada directamente con la supervivencia de los seres humanos, pero el mundo ha sentido de forma abrupta las consecuencias del cambio climático, del cual somos los directos responsables. Estos cambios avanzan de forma vertiginosa, tanto es así que pueden llegar a provocar graves consecuencias como producto del efecto invernadero y el calentamiento global, situaciones de cambios bruscos de las cuales se han encontrado evidencias científicas en la propia historia planeta como resultado de los denominados Ciclos de Milankovitch ${ }^{1}$, y por los cambios en el contenido de $\mathrm{CO} 2$ en la atmósfera ${ }^{2}$. El Ecuador no es la excepción, ante estos eventos y fenómenos, pues el país se encuentra situado sobre el "Cinturón de fuego del Pacifico", zona que se caracteriza por su gran actividad volcánica y sísmica, que son los principales tipos de fenómenos naturales en la lista de eventos que ocasionan los mayores estragos en el hábitat del planeta por su alta ocurrencia y el aumento en sus niveles, causando graves catástrofes en las áreas donde se producen, más aun cuando estos se podrían repetir de una forma consecutiva entre el periodo 2000-2020, con la liberación de niveles de energía con los picos

1 Ciclos de Milankovitch: Pequeñas variaciones en la orientación de la Tierra con respecto al Sol que afectan la manera en que se calienta el planeta. (Caballero, Lozano, \& Beatriz, 2007, pág. 9)

2 Efecto invernadero: mecanismo por el cual la atmósfera de la Tierra se calienta. Ibíd. pág. 3 
más altos en dicho periodo, tal y como lo señala en uno de sus artículos la Universidad Técnica de Panamá (Moncayo, Vargas, Moncayo, \& Barzola, 2017).

En relación con este tema se puede también acotar que, frente a nuestras costas, aproximadamente unos $50 \mathrm{~km}$ de distancia, convergen las placas tectónicas Sudamericana y la de Nazca, frente a la costa norte y centro de Chile y todo el litoral de Perú, Ecuador y Colombia, las mismas que debido al proceso de subducción han producido fuertes sismos con magnitudes muy altas, tal como sucedió en el 2007 en el Perú provocando un terremoto de 7,9 grados en la escala Richter, eventos que pueden provocar tsunamis y a su vez agravar los efectos catastróficos en las poblaciones aledañas a los puertos marítimos, y como no señalar el sismo de 7,8 grados que produjo el terremoto en Ecuador con epicentro en las provincias de Manabí y Esmeraldas el 16 de abril de 2016. Se puede también señalar que en los registros históricos más recientes del país, especificamente en 1906 se produjo un fuerte sismo frente a las costas ecuatorianas y posterior tsunami que prácticamente devasto la provincia de Esmeraldas como se apunta en el reporte "Riesgos por tsunami en la costa ecuatoriana" (Cruz, Acosta, \& Vásquez, s.f.).

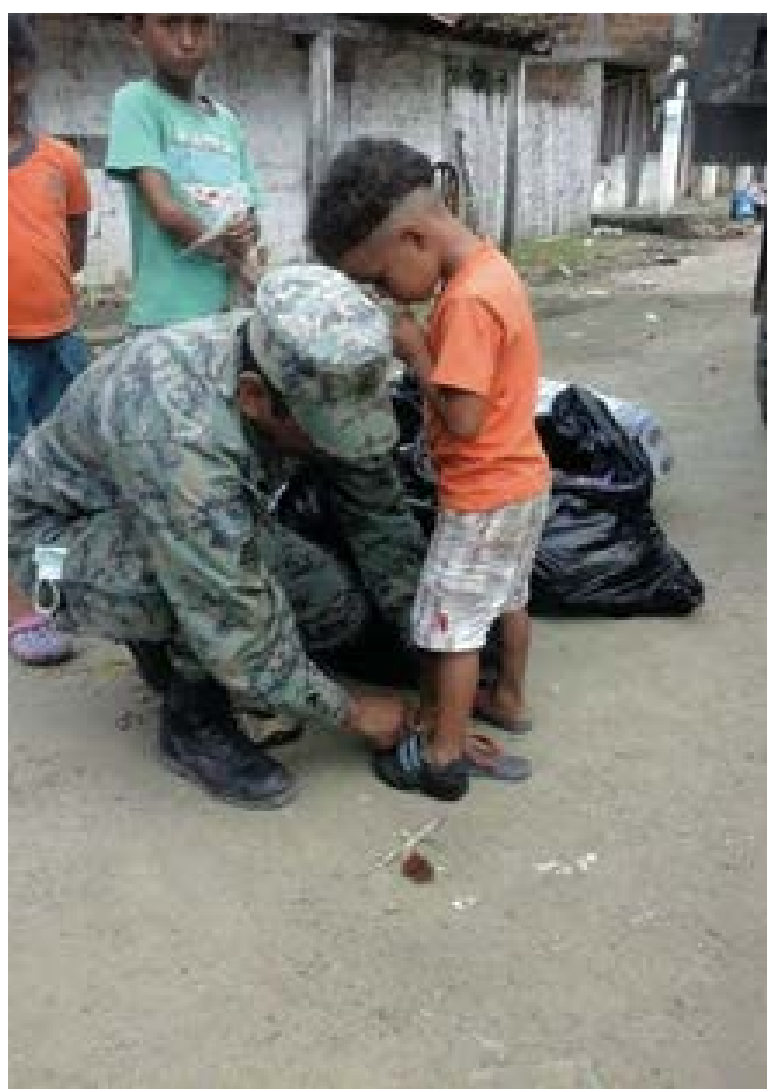

Soldado ecuatoriano proporcionando ayuda a la población afectada en Pedernales en el terremoto del 16 de abril de 2016.
Otros de los estragos a los cuales se ha enfrentado nuestro país son las consecuencias derivadas de las influencias climáticas, para lo cual se ha investigado el artículo "El Ecuador al cruce de varias influencias climáticas. Una situación estratégica para el estudio del fenómeno El Niño", como sucedió con el Niño en 1982-1983 en el cual según (Pourrut, 1998, pág. 452), es de suma importancia que el país cuente con las suficientes previsiones para poder enfrentar los severos impactos que causan este tipo de eventos, especialmente en nuestro país que por su ubicación geográfica es susceptible a que estos ocurran con mayor frecuencia e intensidad, donde las fuertes lluvias han ocasionado la pérdida de vidas humanas y animales, inundaciones, destrucción de infraestructura pública, privada y agrícola, con efectos directos en la economía del país, y que contrario a las etapas de invierno también se tiene otras con abundante escases, propicias a incendios forestales que se han incrementado de forma abrupta con graves consecuencias al ecosistema y a la vida humana.

A la par del registro histórico de la ocurrencia de estos fenómenos, también se tiene el de la intervención de las Fuerzas Armadas, y en forma particular la participación del Ejército en este tipo de emergencias a nivel nacional, donde nuestra contribución ha sido fundamental para acudir en ayuda a la población damnificada a través de los órganos de respuesta que a su debido momento lo constituyeron la Defensa Civil, la Secretaria de Gestión de Riesgos (SGR) hasta hace unos meses atrás y actualmente el Servicio Nacional de Gestión de Riesgos y Emergencias (SNGRE). Situaciones en las que se ha requerido el accionar armónico y planificado de las instituciones, en el que el componente militar que gracias a sus recursos humanos, logísticos y financieros, sumados a sus capacidades han podido estar al frente de cada una de estas situaciones como institución de primera respuesta, proporcionando el soporte a la población en condiciones de riesgo y también contribuyendo a reducir las vulnerabilidades a las cuales tienen que enfrentarse por los estragos ocasionados por estos eventos.

Si bien es cierto FF.AA. ha actuado más en condición de respuesta y reacción que de forma especializada frente a estas situaciones, pero actualmente se ha planteado su inclusión en el concepto de Seguridad Integral, donde se proyecta un nuevo rol para la institución militar, que va acorde a la política pública de Seguridad y Defensa, la que se dio un paso importante cuando se la propuso en el 2002 con la publicación del "Libro Blanco de la Defensa Nacional", en el cual se trató de "determinar las políticas de defensa ante el nuevo escenario geoestratégico de aquel entonces y a las nuevas amenazas, con el fin de preservar el ordenamiento jurídico, la soberanía, la paz y el bienestar ciudadano" (Sánchez C. , 2013, págs. 53,54). 


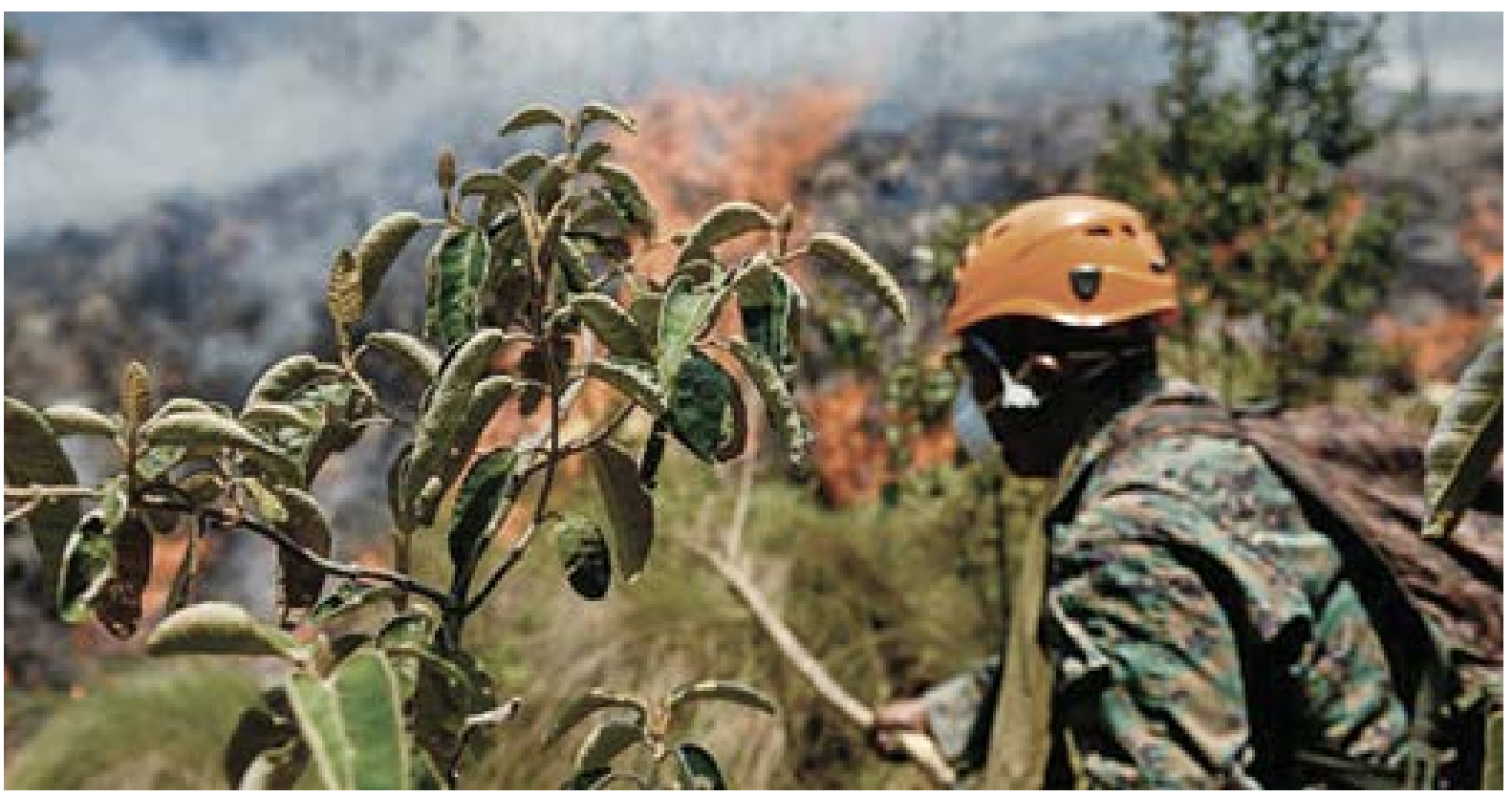

Incendio forestal ocurrido en el cerro Atacazo en Quito y Mejía.

Tomado de: https://www.eltelegrafo.com.ec/noticias/quito/1/aumento-incendios-forestales-quito. www.eltelegrafo.com.ec

Al respecto en el 2003, en la "Conferencia Especial sobre Seguridad de la O.E.A.” que se desarrolló en la ciudad de México, y como una forma de poner atención a las necesidades humanas y sus dramas de seguridad frente a los efectos catastróficos de los eventos naturales y antrópicos que fueron catalogadas dentro de las "nuevas amenazas", como lo señaló Rodríguez ${ }^{3}$, se pone en manifiesto y énfasis el concepto de "seguridad humana"4 y de forma paralela la intervención de las FF.AA. como institución que por su organización, estructura, entrenamiento y medios disponibles, puedan responder frente a estas necesidades humanas colectivas.

Con estos pensamientos de seguridad a nivel mundial en el 2006, se procedió a actualizar el "Libro Blanco" con concepciones de desafío para la seguridad, problemas relacionados al medio ambiente, la sustentabilidad de los recursos naturales, recursos no renovables y otros aspectos más afines (Sánchez C. , 2016, pág. 22), pero estas no pasaron de ser muy generales, sin efectividad de acciones y de decisiones, sin claras proyecciones institucionales para FF.AA. (Celi, 2008, pág. 247), pero ya con la Constitución del 2008 de Montecristi, que posterior a esta se establecieron otros cuerpos legales como la Agenda Nacional de Seguridad Interna y Externa, Ley de Seguridad Pública y del Estado, y el Plan de Seguridad Integral, con los cuales las misiones encomendadas a FF.AA. se han extendido en su dimensión con una idea más amplia de Seguridad Integral, generada sobre la institución más como una demanda histórica (Pérez, 2018, pág. 18), ya con el Plan Nacional de Seguridad Integral 2011-2013 fueron asignadas un número más de tareas relacionadas a la seguridad y defensa nacional, entre estas las de apoyo y colaboración a la Secretaria de Gestión de Riesgos, para hacer frente a los riesgos y desastres, la protección del medio ambiente y su defensa, acciones de ayuda social y otras (Ministerio Coordinador de la Seguridad Interna y Externa, 2011, pág. 38), que luego fueron plasmadas en la Agenda Política de la Defensa 2014-2017, específicamente con la misión de "Apoyar al desarrollo nacional en el ejercicio de las soberanías", como se puede observar en la Ilustración 3, apoyada con el Plan Nacional de Seguridad Integral 2014-2017 con sus respectivas Agendas, entre ellas la "Agenda Sectorial de Gestión de Riesgos", que una de sus partes señala:

Potenciar las capacidades de la sociedad y de sus actores para reducir los niveles de vulnerabilidad. [...]. Desarrolla capacidades para la preparación, prevención, mitigación y reducción de riesgos. Organiza la respuesta humanitaria y asegura

3 El terrorismo, la delincuencia organizada transnacional, [...]. Los desastres naturales y los de origen humano, [...] y el deterioro del medio ambiente. [...] (Rodriguez, 2010, pág. 16).

4 La seguridad humana es la condición necesaria para la subsistencia y calidad de vida de las personas y sociedades, mientras que la seguridad integral, en el campo de la seguridad ciudadana y orden público, constituye una política de Estado destinada a fortalecer y modernizar los mecanismos necesarios para garantizar los derechos humanos [...] (Ministerio Coordinador de la Seguridad Interna y Externa, 2011, págs. 14,15) Revista de la Academia de Guerra del Ejército Ecuatoriano, Volumen 12. Núm. 1 Abril 2019. pp. 103- 108 
que los procesos de reconstrucción erradiquen la vulnerabilidad ${ }^{5}$ (Secretaria de Gestión de Riesgos, 2014, pág. 7).

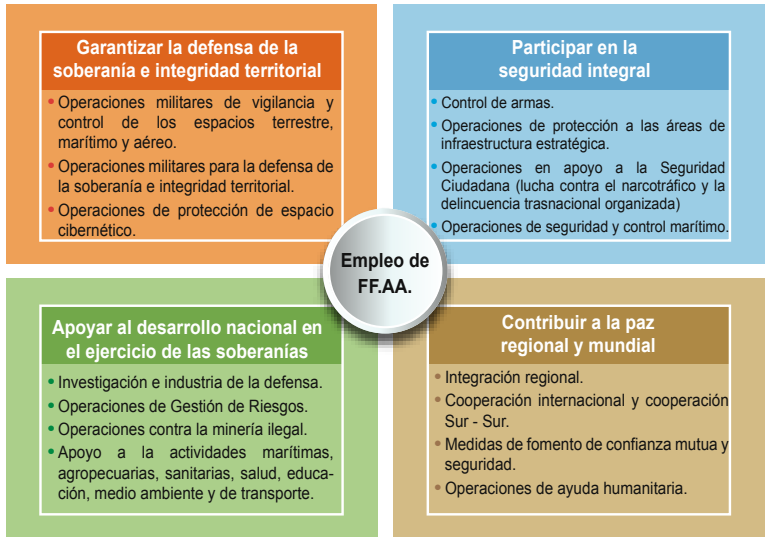

Figura 1. Misiones de Fuerzas Armadas

Fuente: Agenda Política de la Defensa 2014, pág. 40

Ya establecida la gestión de riesgos como parte de la política de seguridad para el Estado ecuatoriano, se pone especial atención en este campo ya que se requiere contar con estrategias que complementen a los Sistemas de Alerta Temprana (SAT) del país, contando como órganos ejecutores de esta política a todas las instituciones públicas y privadas del país, de forma nacional, regional y local (Ministerio de Defensa Nacional, 2014, pág. 21), donde específicamente el Ejército como parte de FF.AA. se constituye en el principal mecanismo operacional terrestre en el territorio para actuar y responder de forma inmediata ante estas situaciones de emergencia nacional, tal y como lo estipula nuestra carta magna (Constitución Política del Ecuador y la enmienda al art. 1586 ; Ley Orgánica de la Defensa Nacional y Ley de Seguridad Pública y del Estado), misión impuesta de forma reciente, aunque esta era cumplida de forma histórica por la institución por encontrarse junto a su pueblo y velar por su seguridad, pero que actualmente lo cumple como parte del apoyo a la seguridad integral impuesta por el Estado, conforme a lo señalado en la política de seguridad y defensa ${ }^{7}$, con el cual se busca emplear de manera óptima todos los recursos que dispone nuestra institución, observándose que:
[..] las demandas sobre la acción militar se amplían, lo que a su vez implica un ámbito expandido de potencial intervención militar, imponiendo presiones sobre la institución en su relación con la sociedad, particularmente a partir de la aprobación de la enmienda constitucional de 2015 mediante la cual su misión fundamental pasa a incorporar el apoyo a la seguridad integral (Pérez, 2018, pág. 17).

En este caso es necesario se puede resaltar que FF.AA. es uno de los principales órganos ejecutores del Sistema de Seguridad Publica y del Estado, institución a la cual se ha dispuesto brinde su apoyo al SNGRE, con todos sus medios, recursos y capacidades disponibles, ante lo señalado anteriormente, en concordancia y cumplimiento a lo dispuesto en el art. 11 y en el literal d) de la Ley de Seguridad Pública y del Estado, los que indican:

Art. 11. - De los órganos ejecutores. - Los órganos ejecutores del Sistema de Seguridad Pública y del Estado estarán a cargo de las acciones de defensa, orden público, prevención y gestión de riesgos, conforme lo siguiente:

d) La prevención y las medidas para contrarrestar, reducir y mitigar los riesgos de origen natural y antrópico o para reducir la vulnerabilidad, corresponden a las entidades públicas y privadas, nacionales, regionales y locales. La rectoría la ejercerá el Estado a través de la Secretaría de Gestión de Riesgos (Asamblea Nacional del Ecuador, 2009, págs. 7,8).

Con respecto al Manual de Gestión de Riesgos se menciona que las FF.AA. tienen funciones asignadas en apoyo directo a los Comités de Operaciones de Emergencia (COE), por lo que deben poner en marcha la respectiva planificación militar para hacer frente de forma anticipada a estas necesidades de seguridad nacional (Secretaría de Gestión de Riesgos, 2014, pág. 41):

1. Apoyar a la Policía Nacional en las acciones para la seguridad de la población y sus bienes, y para la protección de las líneas vitales durante todas las fases de la emergencia.

\footnotetext{
5 La gestión de riesgos es un proceso que los actores públicos y privados llevan a cabo de una manera articulada y de acuerdo con las normas y principios legalmente establecidos para proteger a las personas, colectividades y la naturaleza frente a los efectos negativos de los desastres de origen natural o antrópico mediante la prevención ante el riesgo, la mitigación de desastres, la recuperación y mejoramiento de las condiciones sociales, económicas y ambientales, con el objetivo de minimizar la condición de vulnerabilidad, la necesidad creciente de políticas y mecanismos de articulación y cooperación (Ibíd., pág. 9).

6 "Enmienda Constitucional publicada en el Registro Oficial No. 653 del 21 de diciembre de 2015: Las Fuerzas Armadas tienen como misión fundamental la defensa de la soberanía y la integridad territorial y, complementariamente, apoyar en la seguridad integral del Estado de conformidad con la ley. (Asamblea Nacional del Ecuador, 2015).

7 Las FF.AA., asumen nuevos roles [...], mediante acciones concretas en apoyo a la seguridad interna y orden público, colaboración en la
} gestión de riesgos y desastres, [...] (Ministerio de Defensa Nacional, 2014, pág. 24). 
2. Mantener el orden en coordinación con la Policía Nacional en los lugares afectados.

3. Apoyar la evacuación, búsqueda y rescate de las personas, y el traslado de provisiones, implementos y personal operativo, en coordinación con los $\mathrm{COE}$ para la atención de emergencias.

4. Apoyar las campañas promovidas por el COE.

Con esa finalidad, en el 2010 las FF.AA. preparo el Plan de Apoyo a la Secretaría Nacional de Gestión de Riegos, en caso de desastres naturales o antrópicos, documento anexo al Plan Militar de Defensa Interna de las FF.AA., en donde se establece la siguiente misión:

Las Fuerzas Armadas a través de los Comandos Operacionales, sin descuidar su misión fundamental, con parte de sus medios y en coordinación con la Secretaría Nacional de Gestión de Riesgos, Comités de Operaciones de Emergencia (COE) y demás organismos gubernamentales, apoyarán a la población civil para enfrentar los efectos producidos por los desastres naturales o antrópicos, en forma permanente, en el territorio ecuatoriano, disminuir las consecuencias de los mismos y proteger a las comunidades afectadas a fin de contribuir al cumplimiento de la misión del Comando Conjunto de las Fuerzas Armadas (Comando Conjunto de las FF.AA., 2010, pág. 3).

De esta manera, la institución tiene la tarea de preparar y entrenar a su personal a fin de que se encuentren prestos para cumplir con la misión encomendada, además no solo se requiere que su personal esté en condiciones, se requieren además de los medios necesarios como equipo, material, tecnología, logística, entre otros, que permitan disponer de unas fuerzas listas y en condiciones de emplearse cuando sean requeridas en cualquier momento, circunstancia y situación. De lo señalado se precisa que el personal disponga de la debida capacitación para intervenir en los diversos tipos de eventos que puedan suscitarse, no solo basta el entrenamiento militar, ya que al no constituirse en su misión fundamental es preciso que estén en condiciones de intervenir a través de una sólida respuesta, de forma rápida y eficiente para minimizar los efectos de los desastres. El ejército ha demostrado actuar con oportunidad y eficacia cuando ha sido requerido, los repartos y su personal han participado en diversas circunstancias de emergencia en el país, como ejemplo podemos resaltar la intervención durante los últimos 30 años, como por ejemplo en la inundación de la Josefina en 1993, el fenómeno del Niño de 1997-1998 y 19821983, la erupción del volcán Guagua Pichincha en 1999, erupciones del volcán Tungurahua desde 1999, inundaciones en gran parte del país en el 2008, proceso eruptivo del volcán Cotopaxi en abril de 2014 (F.A.O, s.f, pág. 2) y el último gran desastre ocasionado por el terremoto del 16 de abril de 2016, además de un sinnúmero de eventos provocados por la mano del hombre.

Frente a estas antiguas y nuevas situaciones de riesgo, la institución debe estar en condiciones de responder de forma inmediata en las próximas 72 horas de suscitado el evento, es por eso que se requiere de unidades especializadas de tipo multipropósito básicas, con capacidad operativa y logística para prestar su contingente, considerando que en la actualidad los problemas de narcotráfico, crimen organizado y delincuencia común están multiplicándose como nuevas amenazas que tampoco deben dejarse de lado. Sobre este aspecto, el actual Ministro de Defensa en años atrás manifestaba con total claridad y proyección de futuro lo siguiente:

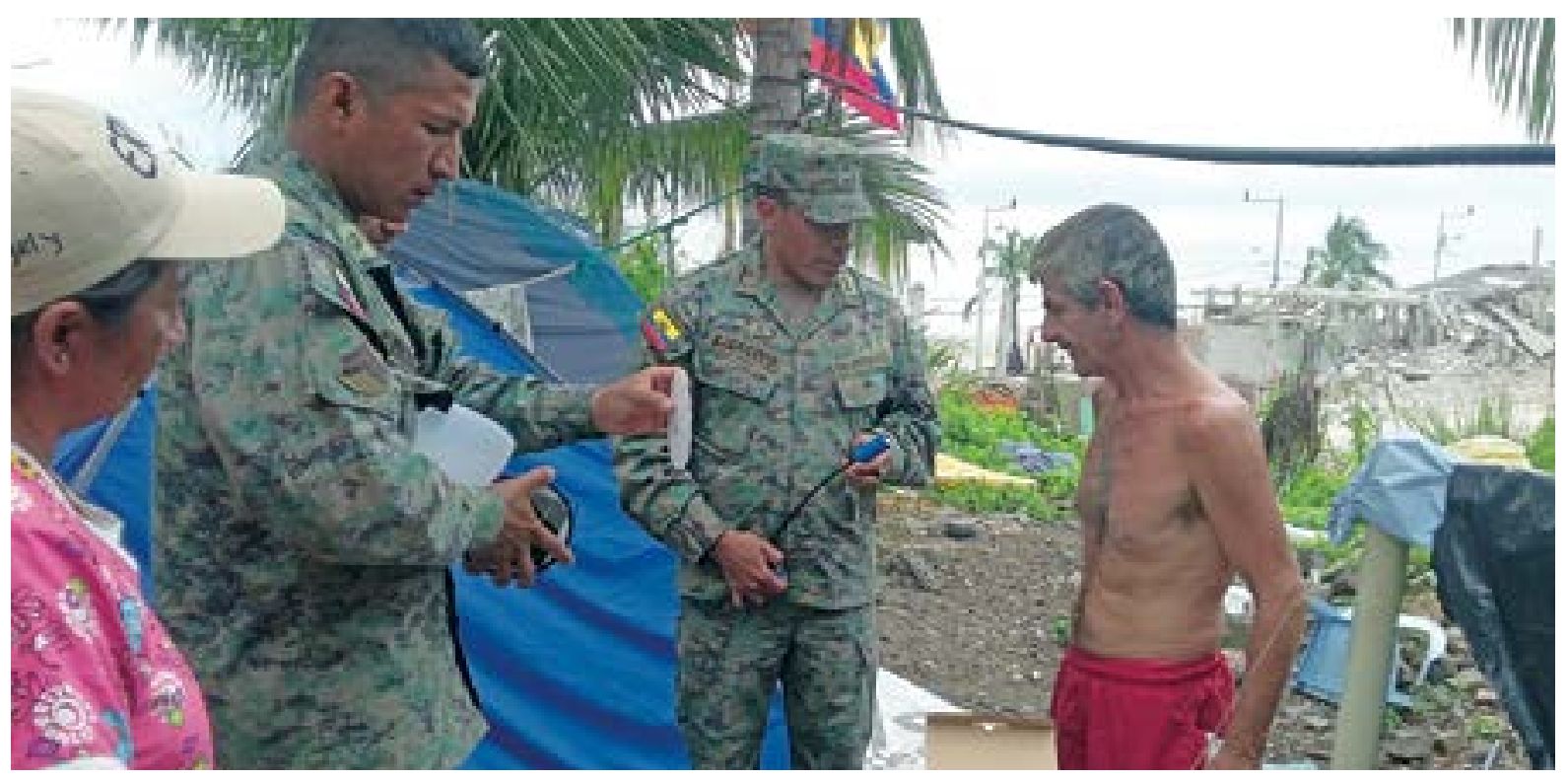

Entrega de ayuda humanitaria a personal civil damnificado por el terremoto del 16 de abril de 2016, habitantes viviendo en carpas en área improvisada junto casa colapsada. 
El país debe tener claro que un proceso de paz no implica el fin del conflicto y el restablecimiento de la seguridad. No va a existir una frontera de paz mientras existan actores armados incontrolables. Son actores que no se rigen por ninguna ley y seguirán actuando. Este proceso de paz con las FARC no tiene nada que ver con el narcotráfico, que seguirá existiendo. El narcotráfico arrastra el resto de delitos conexos, que afectan a la soberanía, convivencia y seguridad del Estado. Por eso, las Fuerzas Armadas no deben desviar el cumplimiento de sus tareas específicas en la frontera (Jarrín, 2012).

Como complemento al tema se debe analizar la posibilidad de contar con unidades debidamente equipadas, entrenadas y capacitadas, que dispongan de medios de despliegue adecuados y oportunos para que cumplan con las actividades de evaluación, evacuación, búsqueda y rescate, primeros auxilios y ayuda de emergencia a los ciudadanos afectados, pues de la presteza con la que se actúe y se de atención a estas situaciones de emergencia, también dependerá la cantidad de vidas humanas que se puedan salvar, cabe señalar el ejemplo de otros países del mundo que han preparado a sus ejércitos para este tipo de situaciones, así tenemos como referentes a los ejércitos de Chile y España que cuentan con repartos especializados y experiencia para este tipo de situaciones, que han obtenido resultados positivos en las diferentes misiones a las cuales han sido destinados.

Haciendo un paréntesis, se puede resaltar que las Unidades Militares de Emergencia en España (UME), fueron creadas el 7 de octubre de 2005, mediante Real Decreto en el cual se dispuso de la configuración, administración y empleo de dichas unidades, como se señala en el texto:

"Las Fuerzas Armadas, junto con las instituciones del Estado y las Administraciones públicas, deben preservar la seguridad y el bienestar de los ciudadanos en los supuestos de grave riesgo, catástrofe, calamidad u otras necesidades públicas, conforme a lo establecido en la legislación vigente" (Ministerio de Defensa de España, 2011).

Al mismo tiempo, se puede resaltar que son unidades es que han intervenido en apoyo a las autoridades españolas para la protección de su población, que también, en otras circunstancias han proporcionado su contingente en otras regiones del mundo, además gracias a su experiencia han creado el Plan de Formación de Unidades Militares de Emergencias (FORUME) para la capacitación de ejércitos de otros países del mundo.

En este aspecto y de forma integradora, la institución está buscando fortalecer sus procesos de actuación, pues no debemos dejar de lado uno de los aspectos de mayor importancia y trascendencia, y en especial considerar que el ejército es parte de nuestro pueblo, conformado por sus propios ciudadanos y que son ellos a los cuales nos debemos, pero en especial resaltar la confianza que ha depositado en nuestras manos de ser los garantes de su seguridad, pues así lo demuestran las encuestas realizadas por CEDATOS (http://www. cedatos.com.ec/detalles_noticia.php? $\mathrm{Id}=367$ ), en las cuales describen a FF.A $\bar{A}$. como la institución de mayor confianza y credibilidad por parte de los ecuatorianos con un porcentaje de aprobación del $80,6 \%$ al 5 de mayo de 2018, distinción que debe ser considerada para fortalecernos al interior y ponerla al servicio de los conciudadanos.

Con este antecedente, se debe estudiar la posibilidad de restructurar a FF.AA. sobre la base de una Planificación por capacidades ${ }^{8}$ para brindar una adecuada acción de apoyo al Estado, tomando de referencia determinados lineamientos planteados por el CC.FF.AA.: 1) Adecuar la organización actual al número de efectivos orgánicos disponibles; 2) Armonizar las normas y procesos operativos para su empleo; 3) Consolidar estructuras modulares multipropósito, con capacidad de insertarse a los sistemas operativos de empleo, en la localidad geográfica en la que se requieran sus acciones; 4) Disponer de un sistema logístico integral que permita satisfacer las necesidades de abastecimiento, mantenimiento y transporte; y 5) Disponer de unidades técnicas de apoyo a la gestión de riesgos y con capacidad de desplazamiento a nivel nacional (Comando Conjunto de las FF.AA., 2011).

Concomitante a lo anterior señalado, las FF.AA. como parte del Sistema de Seguridad Integral del país, tiene la necesidad de fortalecer los procesos de coordinación con las demás instituciones del Estado y de forma particular con el SNGRE (antes SGR) al formar parte de las diferentes Mesas Técnicas de Trabajo de los CGR/COE, encargados de integrar y coordinar las capacidades técnicas de los sectores público y privado, así como también en las actividades específicas de FF.AA. en dichas mesas, las mismas que se detallan en el Manual de Gestión de Riesgos.

8 Planeamiento por capacidades en términos militares, es el conjunto de factores (sistemas de armas, infraestructura, personal y medios de apoyo logístico) asentados sobre la base de unos principios y procedimientos doctrinales que pretenden conseguir un determinado efecto militar a nivel estratégico, operacional o táctico para cumplir las misiones asignadas (Garcia, 2006, pág. 38). 
Al respecto, en la actualidad la institución no se encuentra en las mejores condiciones ya que sus capacidades operativas están reducidas, pero se debe tener presente que lo que se dispone pertenecen a los Sistemas Operacionales del Campo de Batalla (SOCB) para operaciones convencionales con determinadas capacidades que si son debidamente atendidas, fortalecidas y equipadas acorde a las necesidades de apoyo para la gestión de riesgos, pueden mejorar su condición y de esta forma responder ante las situaciones de emergencia, para lo cual se puede destacar como elementos humanos de intervención a través de las unidades subordinadas de la Brigada de Fuerzas Especiales No 9 "PATRIA" (9 B.F.E), que con la movilidad adecuada sea esta terrestre, aérea o fluvial a través de la Brigada de Aviación del Ejército
N 15 "PAQUISHA" (15 B.A.E) y los Comandos Logísticos Regionales (C.L.R), con el soporte y apoyo técnico proporcionados por instalaciones del Hospital de Especialidades $\mathrm{N}^{\circ} 1$ de FF.AA. (H.E 1) y del Cuerpo de Ingenieros del Ejército (C.E.E), con el puntal tecnológico proporcionado por las unidades del Agrupamiento de Comunicaciones y Guerra Electrónica del Ejército (AGRUCOMGE) y apoyo logístico indispensable facilitado por los Comandos Logísticos Regionales (C.L.R) en cada jurisdicción, la institución estaría en condiciones de cumplir con la misión impuesta por el Estado ecuatoriano.

\begin{tabular}{|c|c|}
\hline UNIDADES & TAREAS \\
\hline Cuerpo de Ingenieros del Ejército. & $\begin{array}{l}\text { - Remoción de escombros. } \\
\text { - Proporcionar energía eléctrica de emergencia. } \\
\text { - Construcción de albergues temporales y permanentes. } \\
\text { - Instalar y operar puntos de agua. } \\
\text { - Apoyar en el restablecimiento de servicios básicos. } \\
\text { - Lanzar puentes de circunstancia de hasta } 60 \mathrm{mts} \text {. }\end{array}$ \\
\hline $\begin{array}{l}\text { Agrupamiento de Comunicaciones y Guerra } \\
\text { Electrónica. }\end{array}$ & $\begin{array}{l}\text { - Instalar y operar centros de mando y control. } \\
\text { - Materializar sistemas de comunicación radio (HF, VHF, UHF, Satelital). } \\
\text { - Integración de comunicaciones militares con sistemas de comunicación públicos y } \\
\text { privados. }\end{array}$ \\
\hline $\begin{array}{l}\text { Brigada de Aviación de Ejército N } 15 \\
\text { "PAQUISHA". }\end{array}$ & $\begin{array}{l}\text { - Proporcionar transporte aéreo de personal, equipo y vituallas. } \\
\text { - Ejecutar evacuaciones Aero médicas. } \\
\text { - Realizar búsqueda y rescate de personal y material. } \\
\text { - Ejecutar reconocimientos. }\end{array}$ \\
\hline $\begin{array}{l}\text { Brigada de Fuerzas Especiales N } 9 \\
\text { "PATRIA". }\end{array}$ & $\begin{array}{l}\text { - Búsqueda y rescate se personas sepultadas vivas. } \\
\text { - Rescate de personas aisladas por inundaciones. } \\
\text { - Búsqueda y rescate de montaña } \\
\text { - Seguridad y patrullaje de poblaciones y áreas de riesgo. }\end{array}$ \\
\hline Hospital Quirúrgico Móvil. & $\begin{array}{l}\text { - Proporcionar atención médica integral en apoyo a las operaciones de gestión de riesgos } \\
\text { en las áreas de: consulta externa, emergencias, hospitalización y cirugías mayores. }\end{array}$ \\
\hline Comandos Logísticos Regionales. & $\begin{array}{l}\text { - Almacenar y distribuir abastecimientos. } \\
\text { - Proporcionar transporte terrestre de personal, equipo y vituallas. } \\
\text { - Proporcionar abastecimientos CLASE I (alimentación). } \\
\text { - Organizar, materializar y administrar campamentos y áreas de vivac. }\end{array}$ \\
\hline
\end{tabular}

Fuente: Comando de Operaciones Terrestre de la F.T.

Elaboración: Propia 
Finalmente, las FF.AA. a lo largo de su historia republicana, a más de cumplir con la misión constitucionalmente impuesta por el Estado ecuatoriano ha sido parte fundamental en el desarrollo y principalmente en la seguridad del país, como fue señalado a lo largo de este artículo, es a partir de la conferencia sobre la seguridad desarrollada en México en el 2003 que se dio mayor importancia a la seguridad humana como parte de la seguridad integral, y dentro de esta, considerada su intervención ante la ocurrencia de eventos adversos sean estos de origen natural o antrópico, apoyados con el marco legal y considerando las nuevas amenazas y riesgos que se desarrollan a lo largo del territorio nacional, es indispensable que FF.AA se encuentren debidamente preparadas para enfrentar estos retos que no son nuevos para la institución ya que históricamente se han constituido en el referente de respuesta al frente de las instituciones del Estado, que hoy día requieren de una participación más coordinada ya que este tipo de fenómenos están en aumento y que sumado al crecimiento poblacional también crecen las necesidades de atención que pueden ser asumidas de forma eficiente por unidades especializadas con medios, recursos y entrenamiento específico, esto considerado como aspecto que a la larga demandaría también una reducción en los costes de vidas humanas frente a los de su conformación ya que cuando estos eventos se susciten se podrá intervenir con mayor asertividad y reduciendo los efectos de los desastres.

\section{REFERENCIAS}

[1] Asamblea Nacional del Ecuador. (2009). Ley de Seguridad Pública y del Estado. En Registro Oficial N³5, del 28/09/2009. Quito, Pichincha, Ecuador: Asamblea Nacional del Ecuador.

[2] Asamblea Nacional del Ecuador. (2015). Registro Oficial No. 653. Dic. del 2015. Quito, Pichincha, Ecuador.

[3] Caballero, M., Lozano, S., \& Beatriz, O. (2007). Efecto Invernadero, Calentamiento Global y Cambio Climático: Una perspectiva desde las ciencias de la Tierra. Recuperado el 08 de mayo de 2018, de Revista Digital Universitaria $\mathrm{N}^{\circ} 10$, Vol. 8: http:// www.revista.unam.mx/vol.8/num10/ art78/oct_art78.pdf

[4] Celi, P. (2008). La seguridad y la defensa en una nueva perspectiva». La Tendencia: Análisis Nueva Constitución. En FlacsoAndes, \& ILDIS, Análisis: Nueva Constitución (págs. 245 - 263). Quito, Pichincha, Ecuador.

[5] Comando Conjunto de las FF.AA. (2010). "Plan de Apoyo de las Fuerzas Armadas a la Secretaría Nacional de Gestión de Riesgos, en caso de desastres naturales o antrópicos". Quito, Pichincha, Ecuador.

[6] Comando Conjunto de las FF.AA. (2011). Directiva $N^{\circ} 2$ para la Reestructuración de FF.AA. Quito, Pichincha, Ecuador.

[7] Cruz, M., Acosta, M., \& Vásquez, N. (s.f.). "Riesgos por Tsunami en la Costa Ecuatoriana. Recuperado el 15 de abril de 2018, de Instituto Panamericano de Geografía e Historia.: http://www.ipgh.gob.ec/ documentos/recursos/Riesgos $\% 20$ Tsunami $\% 20$ Costa $\% 20$ ecuatoriana.pdf
[8] F.A.O. (s.f). Desastres naturales y tenencia de tierras. Recuperado el 18 de abril de 2018, de Tierra segura: http://www.fao.org/docrep/013/i1255b/ i1255b02.pdf

[9] Garcia, J. (Junio de 2006). Planeamiento por Capacidades. Recuperado el 15 de marzo de 2018, de Revista Española de Defensa: http://www.defensa.gob.es/Galerias/ documentacion/revistas/2006/220.pdf

[10] Jarrín, O. (16 de sep. de 2012). El proceso de paz en Colombia no elimina la violencia en la frontera. Recuperado el 05 de mayo de 2018, de El Comercio: http://www.elcomercio. com.ec/politica/entrevista-Colombiaproceso_de_paz-Juan_Manuel_SantosOswaldo_Jarrin-FARC__0_774522632. html

[11] Ministerio Coordinador de la Seguridad Interna y Externa. (2011). Plan Nacional de Seguridad Integral 2011-2014. Quito, Pichincha, Ecuador.

[12] Ministerio de Defensa de España. (2011). UME. Recuperado el 10 de 05 de 2018, de Unidad Militar de Emergencias, primera respuesta a la nueva misisón de Fuerzas Armadas: http://www.defensa.gob. es/ume/Galerias/dossier/Descargas/ dossier_2011.pdf

[13] Ministerio de Defensa Nacional. (2014). Agenda Sectorial de Defensa. En MICS, Plan Nacional de Seguridad Integral 2014 - 2017 (pág. 24). Quito, Pichincha, Ecuador.

[14] Moncayo, M., Vargas, J., Moncayo, G., \& Barzola, I. (2017). Análisis sobre la recurrencia de terremotos severos en Ecuador". Recuperado el 01 de mayo de 2018, de Revista digital UTP N¹, vol. 8.: http://revistas. utp.ac.pa/index.php/prisma/article/ view/1526/html
[15] Pérez, D. (2018). Fuerzas Armadas en Ecuador. Ideología, políticas publicas y dialogo civil militar. Quito.

[16] Pourrut, G. (1998). "El Ecuador al cruce de varias influencias climáticas. Una situación estratégica para el estudio del fenómeno de El Niño". Recuperado el 02 de mayo de 2018, de Redalcy; Bulletin de I'Institut Francais d'Etudes Andines: http://www.redalyc. org/articulo.oa?id=12627310

[17] Rodriguez, G. (2010). Antiguas y nuevas amenazas a la Seguridad en América Latina. Recuperado el 27 de abril de 2018, de Fudación Preciado: www.fundacionpreciado.org.mx

[18] Sánchez, C. (2013). Empleo de FF.AA., en el control del orden público y seguridad ciudadana. Tesis de Maestria en Seguridad y Defensa: IAEN. Quito.

[19] Sánchez, C. (2016). Política Pública de Seguridad y Defensa en el Ecuador y el nuevo rol de Fuerzas Armadas. En A.G.E., Boletín Académico de la Academia de Guerra del Ejército (pág 22). Quito, Pichincha, Ecuador.

[20] Secretaria de Gestión de Riesgos. (2014). Agenda Sectorial de Gestión de Riesgos. En MICS, Plan Nacional de Seguridad Integral 2014-2017'. (pág. 7). Quito, Pichincha, Ecuador.

[21] Secretaría de Gestión de Riesgos. (2014). Manual de Gestión de Riesgos. Quito, Pichincha, Ecuador. 